\title{
Formation of regular nanoscale undulations on a thin polymer film imprinted by a soft mold
}

\author{
Kahp Y. Suh ${ }^{\text {a) }}$ \\ School of Mechanical and Aerospace Engineering, Seoul National University, Seoul 151-742, Korea \\ Soon Min Seo, Pil J. Yoo, and Hong H. Lee \\ School of Chemical and Biological Engineering, Seoul National University, Seoul 151-742, Korea
}

(Received 19 October 2005; accepted 15 November 2005; published online 10 January 2006)

\begin{abstract}
We observed the formation of regular nanoscale undulations on a polystyrene film when imprinted by a soft poly(dimethylsiloxane) mold above the polymer's glass transition temperature. The shape of the wave was reminiscent of a buckling wave frequently observed for a metal film supported on an elastomeric substrate. We derived a simple theoretical model based on an anisotropic buckling of the polymer film rigidly bound to a substrate, which agrees well with the experiment. (C) 2006 American Institute of Physics. [DOI: 10.1063/1.2150211]
\end{abstract}

\section{INTRODUCTION}

Nanoimprint lithography (NIL) is a low-cost method for generating resist relief patterns in a thermoplastic layer by physically compressing the resist that has been thermally softened, rather than by modifying the resist's chemical structure by radiation. ${ }^{1}$ Since NIL typically involves a hightemperature process above the polymer's glass transition temperature $\left(T_{g}\right)$, various types of self-assembled structures have been reported based on electrostatic interactions ${ }^{2}$ or van der Waals forces. ${ }^{3,4}$ These instabilities would be detrimental to the optimization of NIL process particularly as the feature size becomes smaller and the stamp geometries become more complex. In parallel, the spontaneous pattern formation would be potentially of interest for micro- or nanoscale lithography.

Recently, we developed capillary force lithography ${ }^{5}$ (CFL) by combining the essential feature of NIL of molding a polymer melt with the prime element of microcontact printing or soft lithography of using an elastomeric mold such as poly(dimethylsiloxane) (PDMS). As a result, the advantage of NIL over the microcontact printing is retained in meeting the stringent pattern fidelity requirements for fabricating integrated circuits while eliminating the need to use an extremely high pressure that is needed in NIL. In CFL, a patterned PDMS mold is placed on a spin-coated polymer film and then heated above $T_{g}$, such that the capillarity forces the polymer melt into the void space of the mold, thus forming a negative replica after mold removal. CFL is also a hightemperature process and under certain conditions generates an ordered, dewetting structure around the protruding feature in contact with the polymer surface particularly when a low molecular weight polymer is used $\left(M_{w}<40000\right) .{ }^{6}$ It is noted in this regard that CFL has less to do with previous instabili-

\footnotetext{
${ }^{a)}$ Author to whom correspondence should be addressed. Electronic mail: sky4u@snu.ac.kr
}

ties based on electrostatic interactions or trapped air within the void space of the mold because PDMS is less charged and probably the most permeable rubber polymer to air. ${ }^{7}$ In order to suppress dewetting in CFL, thin thermoplastic resins such as polystyrene (PS) with a high molecular weight could be used as a robust etch resist for subsequent dry pattern transfer (e.g., reactive ion etching), which turned out to be quite effective. ${ }^{8}$ As the film thickness decreases to less than $100 \mathrm{~nm}$ for decreasing a residual layer, a new type of instability was observed on the surface in contact with the mold even for the high molecular weight polymer, which is a major topic in this study. In this spontaneous pattern formation, a regular, anisotropic wave was formed, which is reminiscent of an anisotropic buckling of a metal layer supported on a polymer film that was reported earlier by the same authors. ${ }^{9}$ Although the undulations are on the order of a few nanometers and thus have minimal effects on the subsequent pattern transfer, it would be of interest to investigate the pattern formation and its potential applications.

Physical self-assembly of microstructures by the anisotropic buckling seems to be an alternative to microshaping a metal surface on a polymer film. ${ }^{9-12}$ In contrast to the chemical self-assembly that exploits chemical interactions between a self-assembled monolayer and a substrate, ${ }^{13}$ physical selfassembly utilizes physical forces such as mechanical stress, ${ }^{9}$ capillarity, ${ }^{14}$ or dispersion forces ${ }^{15}$ to drive the surface into a desirable shape. The buckling-induced wave formation on an organic layer (polymer film in general) supported on a substrate $^{16}$ or covered with a substrate and a capping layer was reported by a number of groups. ${ }^{17,18}$ In order to generate a mechanical stress, temperature was raised above the glass transition or a polymer film was dipped in a poor solvent for swelling. Interestingly, the buckling also takes place for a soft polymer film supported on a rigid substrate in response to a swelling in a poor solvent, ${ }^{18}$ which differs from a conventional buckling for a rigid film coated on a soft substrate. $^{10,19}$ We believe that this buckling is also closely 
related to the wave formation of a thin polymer film rigidly bound to a substrate. As with the anisotropic buckling of a bilayer of metal on polymer, the buckling presented here also occurs in an anisotropic manner mediated by the applied line features of the mold. A potential application of the wave formation is demonstrated here for roughly estimating Young's modulus of a thin polymer film such as PS.

\section{EXPERIMENTAL METHOD}

\section{A. Sample preparation}

We fabricated PDMS (Sylgard 184, Dow Corning) molds that have a planar surface with recessed patterns by casting PDMS against a complementary relief structure prepared by photolithographic method. Two types of PDMS molds were used. The first mold had a line-and-space pattern with the spacing of $1 \mu \mathrm{m}$ and the linewidths of 5, 10, and $15 \mu \mathrm{m}$, respectively. The second mold had an equal line-andspace pattern with the width of $5 \mu \mathrm{m}$. The height of the protruding lines was $1 \mu \mathrm{m}$. A PS of high molecular weight $\left(M_{w}=2.3 \times 10^{5}, M_{w} / M_{n}=1.05\right.$, and $T_{g}=100{ }^{\circ} \mathrm{C}$, Aldrich $)$ was used throughout the experiment. In order to suppress dewetting, silicon substrate was dipped into pirana solution $\left(\mathrm{H}_{2} \mathrm{SO}_{4}: \mathrm{H}_{2} \mathrm{O}_{2}=4: 1\right)$ for $20 \mathrm{~min}$ and then dipped into 10 wt $\%$ HF solution, rendering a hydrogen-terminated surface. Using this substrate, dewetting was strongly suppressed even after annealing the film for $24 \mathrm{~h}$ at $150{ }^{\circ} \mathrm{C}$, suggesting that the film was rigidly bound to the substrate. The PDMS mold was placed on the surface of a polymer layer that was spin coated onto silicon substrate and then the sample was heated well above $T_{g}$ of the polymer (typically $130{ }^{\circ} \mathrm{C}$ ). Prior to mold placement, PS films were annealed in a vacuum oven overnight at $150{ }^{\circ} \mathrm{C}$ to remove any residual solvent. Polymer films were spin coated onto the substrate to $\sim 28-\sim 121 \mathrm{~nm}$ thickness as confirmed by ellipsometry.

\section{B. Atomic force microscopy}

Atomic force microscopy (AFM) images were taken in tapping mode on a NanoScope III Dimension (Veeco Instruments Inc.) in air. The scan rate was $0.5 \mathrm{~Hz}$ and 256 lines were scanned per sample. Tapping mode tips, NSC15 -300 kHz, were obtained from MikroMasch (Portland). Data were processed using NANOSCOPE III 4.31r6 software (Veeco Instruments Inc.). Some of the images were flattened but not further manipulated. To measure a peak wavelength from the cross-sectional profile, the fast Fourier-transformed (FFT) data were obtained from an AFM image processor and then compared with the wavelength that was determined from a topographical analysis of the cross-sectional profile.

\section{Scanning electron microscopy}

Scanning electron microscopy (SEM) images were taken using high-resolution SEM (XL30FEG, Philips Electron Co., Netherlands) at an acceleration voltage of $3 \mathrm{eV}$ and a working distance of $7 \mathrm{~mm}$. The samples were coated with a $30 \mathrm{~nm} \mathrm{Au} \mathrm{layer} \mathrm{prior} \mathrm{to} \mathrm{analysis} \mathrm{to} \mathrm{prevent} \mathrm{charging.}$

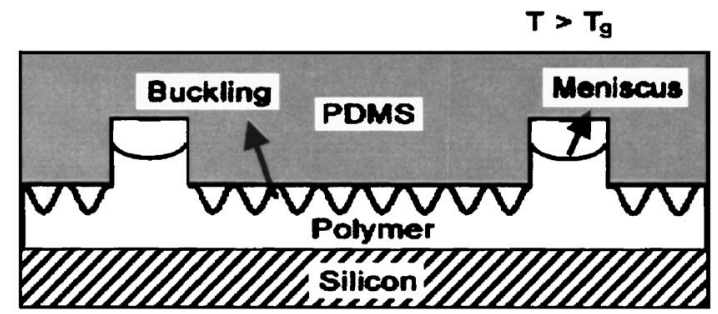

(a)

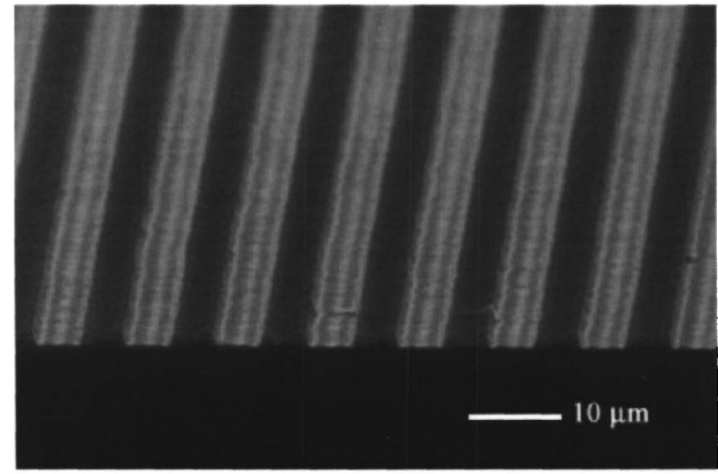

(b)

FIG. 1. (a) A schematic illustration of the experimental setup. (b) A SEM image of the anisotropic wave formation along the line direction when a $1 \mu \mathrm{m}$ line-and-space PDMS mold was applied on a $\sim 100 \mathrm{~nm}$ PS film for $12 \mathrm{~h}$ at $130{ }^{\circ} \mathrm{C}$ (darker regions correspond to the polymer).

\section{RESULTS AND DISCUSSION}

A schematic illustration of the experimental setup is shown in Fig. 1(a). With the PDMS mold in place, without any external force, the temperature was raised above $T_{g}$ of the polymer. After the wave formation took place, the mold was simply removed, finishing the anisotropic pattern formation. Shown in Fig. 1(b) is a typical SEM image of the wave formation along the line direction when a $5 \mu \mathrm{m}$ line-andspace PDMS mold was applied on a $\sim 100 \mathrm{~nm}$ PS film for $12 \mathrm{~h}$ at $130{ }^{\circ} \mathrm{C}$ (darker regions correspond to the polymer). The height was $\sim 190 \mathrm{~nm}$ for the molded region and $\sim 30 \mathrm{~nm}$ for the wave region, respectively. As shown in the figure, a well-defined PS replica was fabricated except for repeating two lines between the adjacent molded PS structures. This gives fundamental cues how the structures were formed during the capillary rise of the polymer. If a single line was left behind between the molded structures, one might assume that the polymer in contact with the mold was not completely removed in part due to physical confinement between the mold and the polymer. The formation of two repeating lines, however, is difficult to describe with a simple mass transport and thus implies that a certain type of wave instability should be responsible.

To elaborate on this wave formation, we tested various linewidths with different film thicknesses. Figure 2(a) shows the AFM images of the surface waves that formed for various film thicknesses with the linewidth fixed at $15 \mu \mathrm{m}$ ( $L$ $=15 \mu \mathrm{m})$. The wave periods were determined by analyzing the surface topography (the distance between the markers divided by the number of peaks) together with the fast Fourier-transformed images in Fig. 2(b). To obtain the spectra, the images were zoomed in inside the wave region be- 


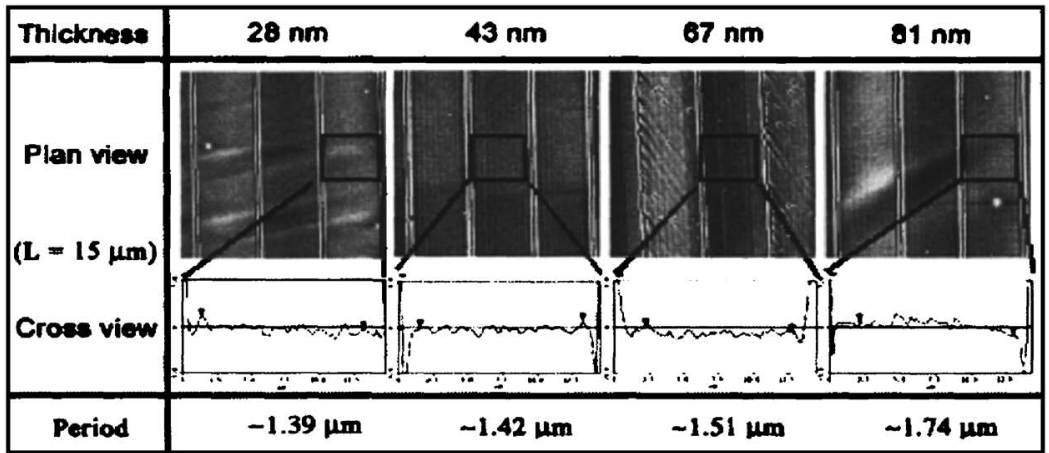

(a)

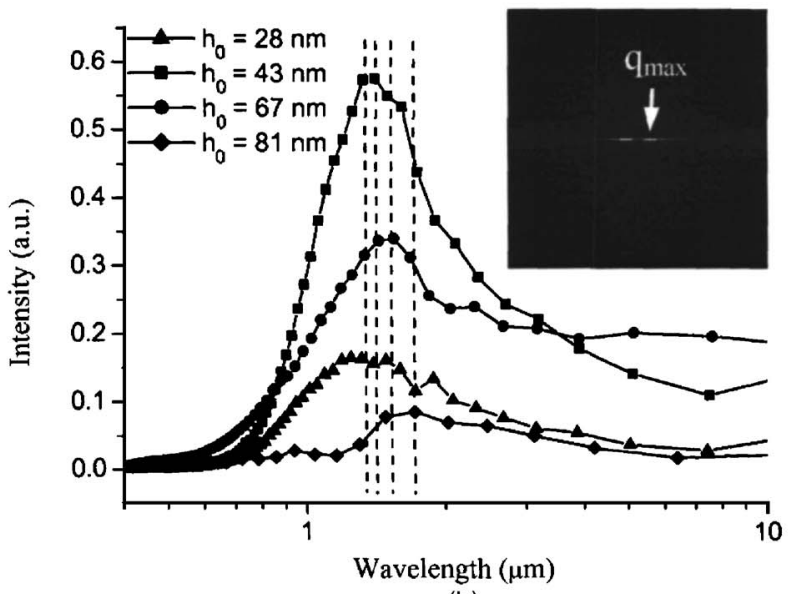

(b) tween the two polymer walls that were induced by capillarity. As the height scale is $10 \mathrm{~nm}$, the polymer walls seem to be infinite boundaries. A typical FFT image is also shown in the inset, which clearly indicates a peak wave vector in the reciprocal space. Apparently, no temporal evolution of the amplitude or the wavelength was observed such that the pattern forms instantaneously and then remains static. The amplitude of the wave appeared to depend on the film thickness of PS, ranging from $\sim 0.7$ to $\sim 1.2 \mathrm{~nm}$ with increasing film thickness. This amplitude is much smaller than that for $1 \mu \mathrm{m}$ linewidth observed in Fig. 1(b), suggesting that the amplitude is related to the initial film thickness and the pattern width. As shown in Figs. 2(a) and 2(b) and explained later, the wavelength slightly increases with increasing film thickness with an exponent much less than 2 for conventional capillary wave theory, suggesting that a different instability mechanism comes into play for the wave formation presented in this study.

When the linewidth becomes smaller, the amplitude increases to $2.5-3 \mathrm{~nm}$ for the $10 \mu \mathrm{m}$ linewidth and $3.2-4.7 \mathrm{~nm}$ for the $5 \mu \mathrm{m}$ linewidth, respectively, with essentially no change in the pattern periods that were measured from the FFT images, as shown in Fig. 3(a). In a series of experiments in which the film thickness was varied with different linewidths, we found that the intrinsic period of the anisotropic wave increases slightly with the thickness such that its exponent ranges from 1.39 to $1.78 \mu \mathrm{m}$, as shown in Fig. 3(b) (the exponent is only 0.3).

The anisotropic wave formation resembles that of a buckling of a metal layer supported on an elastomeric film. More closely, it appears to be related to the buckling of a soft polymer film supported on a rigid substrate in response to a swelling in a poor solvent ${ }^{18}$ or an elastic instability of rubber films sandwiched between two solid bodies. ${ }^{20}$ A qualitative explanation is that the PS film experiences a compressive stress when cooling down to room temperature because it is confined vertically by the imposed PDMS mold and the substrate. Furthermore, capillary flow into a mold cavity generates a compressive stress in the film in contact with the mold. The difference from the conventional buckling system (a metal layer supported on an elastomeric film) is that the polymer film is supported on a rigid substrate while the elastomeric PDMS imposes a physical barrier along with the nodal condition. In other words, the imposed PDMS mold not only generates an anisotropic buckling wave, but also hinders the growth of a surface wave once it is generated, leading to very small amplitudes on the order of a few nanometers. In our experiment, the nodal condition is given by

$$
L=\frac{2 n-1}{2} \lambda \quad(n=1,2,3, \ldots),
$$

where $L$ is the channel width and $\lambda$ is the wavelength. Comparison of the wave periods with the channel widths in Fig. 3(b) showed that in contrast to the buckling of a metal film, the nodal condition was not strictly satisfied, rendering the value of $n$ of 3 or 4 . As the polymer film is not purely elastic, some viscoelastic effects might be responsible for this behavior. 
(a)
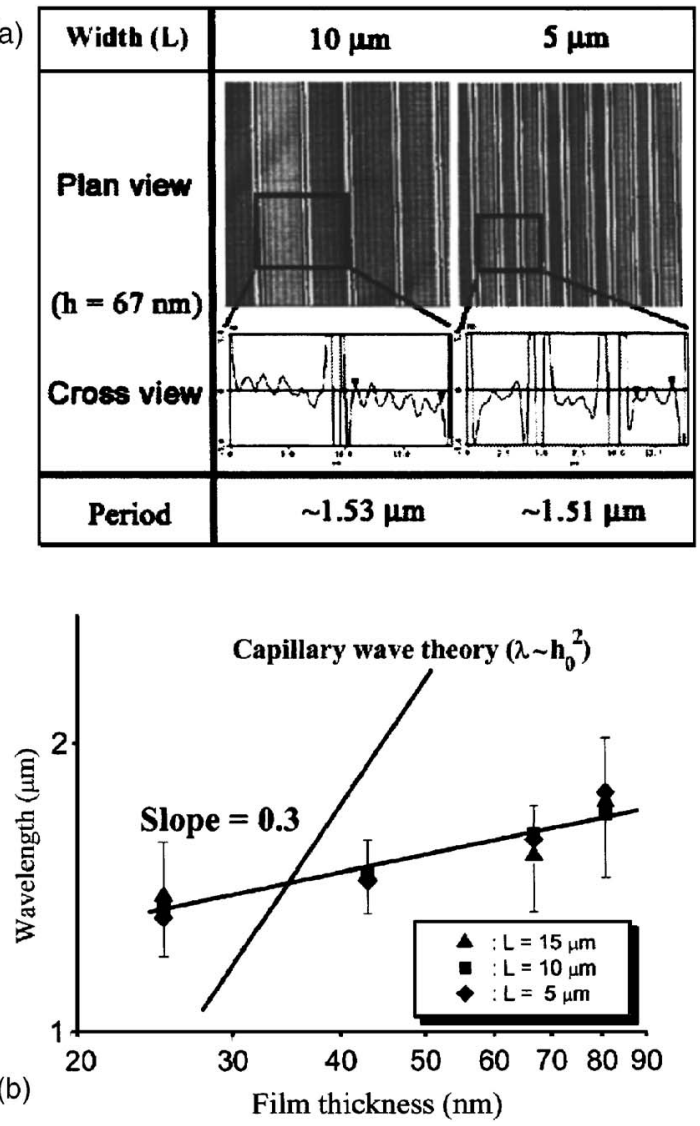

FIG. 3. (a) Planar and cross-sectional AFM micrographs of the surface waves that formed for 67-nm-thick film at given linewidths (5 and $10 \mu \mathrm{m})$. The $z$ scale corresponds to $10 \mathrm{~nm}$. (b) Dependence of the wavelength of the anisotropic buckling wave on the initial film thickness.

From an application point of view, this wave formation would have limited utility for fabricating polymer microstructures or nanostructures. For one, the amplitude generated on the surface is merely on the order of a few nanometers and the surface does not show excellent pattern fidelity nor regularity. Nonetheless, this simple buckling could be useful in roughly estimating Young's modulus of the thin polymer film as described below.

A traditional approach to dealing with buckling is to consider the balance between the swelling stress (thermal stress in this case) in the unbuckled film and the bending stress in the buckled state, and the forces required to overcome the adhesion of the film to the substrate. ${ }^{18}$ For the experiment presented here, the amplitude is merely on the order of a few nanometers such that the bending stress appears to be negligible. Furthermore, the competition between the swelling and bending stresses results in the linear dependence of the buckling wavelength on the film thickness, which does not agree with our experiment. Thus, the swelling stress should be counterbalanced by a resisting force that prevents the formation of a surface wave, which reduces to a simple instability theory for the competition between thermal stress and surface tension. For a theoretical description of the ripple instability, it is sufficient to consider the energy minimization since the pattern formation is static. According to a

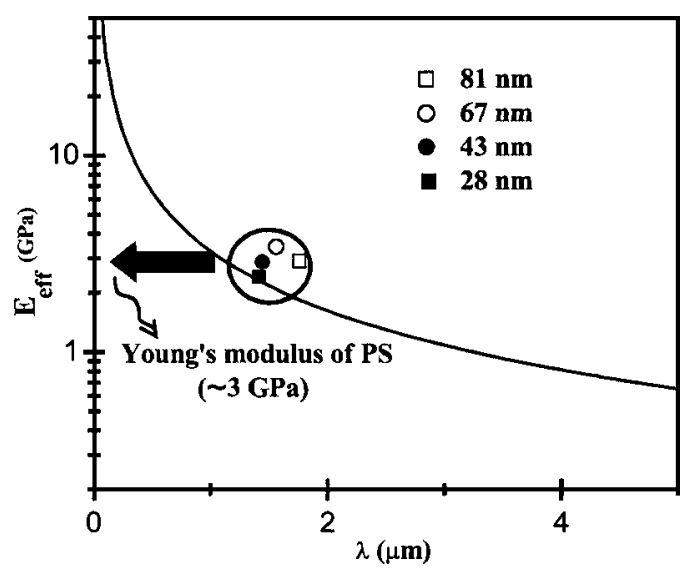

FIG. 4. Dependence of the effective Young's modulus $\left(E_{\text {eff }}\right)$ on the wavelength $(\lambda)$. The symbols indicate the experimental data measured using four different film thicknesses. Note that the modulus is in good agreement with the bulk value in the literature ( $\sim 3 \mathrm{GPa}$ (Ref. 22$)$ ).

previous study, ${ }^{21}$ the fastest growing wave number, $q_{\max }$, in the absence of the contribution from the intermolecular forces is given by

$$
q_{\max }^{2}-\frac{3 \sigma_{0}^{2}}{2 E \gamma} q_{\max }=0,
$$

where $\sigma_{0}$ is the built-in thermal stress, and $\gamma$ and $E$ are the surface tension and Young's modulus of the film, respectively. As was reported earlier, $q_{\max }$ becomes independent of the thickness if the stress term dominates over the dispersion term, such that the power index for the thickness dependence becomes zero. Although the exponent observed in our study slowly increases with increasing film thickness or a contribution from the intermolecular forces cannot be completely excluded, we assume that the pattern periods are mainly determined by anisotropic buckling and not by other instabilities for mathematical simplicity. Upon cooling to room temperature, the polymer film experiences a compressive stress that is given by

$$
\sigma_{0}=\int_{T_{0}}^{T_{g}} \frac{E}{1-\nu}\left(\alpha_{f}-\alpha_{s}\right) d T \approx\left(\frac{E_{\mathrm{eff}}}{1-\nu}\right)\left(\alpha_{f}-\alpha_{s}\right)\left(T_{g}-T_{0}\right),
$$

where $T_{0}$ is the stress-free, initial temperature, $\nu$ is the Poisson ratio, and $\alpha_{s}$ and $\alpha_{f}$ are the thermal-expansion coefficients of the substrate and the film, respectively. Here, $E_{\text {eff }}$ is the effective Young's modulus that can explain the weak temperature dependence of Young's modulus for the glassy state. Combining Eqs. (1) and (2) leads to a relation between the wavelength $(\lambda)$ and $E_{\text {eff }}$, which is given by

$$
E_{\text {eff }}=\frac{4 \pi \gamma(1-\nu)^{2}}{3 \lambda\left(\alpha_{f}-\alpha_{s}\right)^{2}\left(T_{g}-T_{0}\right)^{2}} .
$$

Shown in Fig. 4 is the plot of the effective Young's modulus of PS film as a function of the wavelength. For the plot, the following values were used: $\gamma=40 \mathrm{~mJ} / \mathrm{m}^{2}, \nu=0.3$, $\alpha_{f}=6.7 \times 10^{-5}{ }^{\circ} \mathrm{C}^{-1}, \alpha_{s}=5.5 \times 10^{-7}{ }^{\circ} \mathrm{C}^{-1}, T_{g}=100^{\circ} \mathrm{C}$, and $T_{0}=25^{\circ} \mathrm{C}^{22}$ The symbols in the figure indicate the experimental data at four different film thicknesses. As shown in 
the figure, the effective Young's modulus decreases monotonically with increasing period of the wave. Interestingly, the data are scattered within the narrow window as marked with the circle in the figure, which are in good agreement with the bulk values in the literature $(\sim 3 \mathrm{GPa}) .^{22}$ This result shows that Young's modulus of the underlying polymer film in glassy state might be estimated by simply analyzing the depth profile of the resulting wave.

\section{SUMMARY}

An anisotropic buckling wave has been observed for a thin polymer supported on a rigid substrate when a soft, patterned PDMS mold was imposed on the surface. The period of the surface wave was measured for various film thicknesses by analyzing the cross-sectional profiles of AFM images and FFT spectra. It turned out that the wavelength slightly increased with increasing film thickness with the power index of 0.3 , suggesting that a new instability based on mechanical stress governs the phenomenon via anisotropic buckling. A future study would address the feasibility of applying our approach to other polymer films.

\section{ACKNOWLEDGMENT}

This work was supported by the Micro Thermal Research Center of Seoul National University.
${ }^{1}$ S. Y. Chou, P. R. Krauss, and P. J. Renstrom, Science 272, 85 (1996).

${ }^{2}$ S. Y. Chou, L. Zhuang, and L. J. Guo, Appl. Phys. Lett. 75, 1004 (1999).

${ }^{3}$ H. Schift, L. J. Heyderman, M. A. D. Maur, and J. Gobrecht, Nanotechnology 12, 173 (2001).

${ }^{4}$ L. J. Heyderman, H. Schift, C. David, J. Gobrecht, and T. Schweizer, Microelectron. Eng. 54, 229 (2000).

${ }^{5}$ K. Y. Suh, Y. S. Kim, and H. H. Lee, Adv. Mater. (Weinheim, Ger.) 13, 1386 (2001).

${ }^{6}$ K. Y. Suh, J. Park, and H. H. Lee, J. Chem. Phys. 116, 7714 (2002).

${ }^{7}$ T. C. Merkel, V. I. Bondar, K. Nagai, B. D. Freeman, and I. Pinnau, J. Polym. Sci., Part B: Polym. Phys. 38, 415 (2000).

${ }^{8}$ K. Y. Suh and H. H. Lee, Adv. Funct. Mater. 12, 405 (2002).

${ }^{9}$ P. J. Yoo, K. Y. Suh, S. Y. Park, and H. H. Lee, Adv. Mater. (Weinheim, Ger.) 14, 1383 (2002).

${ }^{10}$ N. Bowden, S. Brittain, A. G. Evans, J. W. Hutchinson, and G. M. Whitesides, Nature (London) 393, 146 (1998).

${ }^{11}$ P. J. Yoo, S. Y. Park, S. J. Kwon, K. Y. Suh, and H. H. Lee, Appl. Phys. Lett. 83, 4444 (2003).

${ }^{12}$ T. Okayasu, H. L. Zhang, D. G. Bucknall, and G. A. D. Briggs, Adv. Funct. Mater. 14, 1081 (2004).

${ }^{13}$ Y. N. Xia and G. M. Whitesides, Annu. Rev. Mater. Sci. 28, 153 (1998).

${ }^{14}$ K. Y. Suh and H. H. Lee, Adv. Mater. (Weinheim, Ger.) 14, 346 (2002).

${ }^{15}$ G. Reiter, Phys. Rev. Lett. 68, 75 (1992).

${ }^{16}$ M. Muller-Wiegand, G. Georgiev, E. Oesterschulz, T. Fuhrmann, and J. Salbeck, Appl. Phys. Lett. 81, 4940 (2002).

${ }^{17}$ K. Dalnoki-Veress, B. G. Nickel, and J. R. Dutcher, Phys. Rev. Lett. 82, 1486 (1999).

${ }^{18}$ J. S. Sharp and R. A. L. Jones, Adv. Mater. (Weinheim, Ger.) 14, 799 (2002).

${ }^{19}$ H. G. Allen, Analysis and Design of Structural Sandwich Panels (Pergamon, Oxford, 1969).

${ }^{20}$ W. Monch and S. Herminghaus, Europhys. Lett. 53, 525 (2001).

${ }^{21}$ K. Y. Suh and H. H. Lee, Phys. Rev. Lett. 87, 135502 (2001).

${ }^{22}$ J. Brandup and E. H. Immergut, Polymer Handbook (Wiley, New York, 1989). 